\title{
Anti-PUE Attack Base on Fractal Dimension in Spectrum Sensing
}

\author{
$\mathrm{Xu}$ Jie and Fu Shuang \\ College of Information Technology, Heilongjiang Bayi Agricultural University, \\ Daqing, 163319 China \\ byndxj@163.com,fushuang_dq@163.com
}

\begin{abstract}
Secure problem has become a major concern in spectrum sensing. PUE attack is a common attack in spectrum sensing. To defend PUE attack, an anti-PUE attack method in spectrum sensing based on fractal dimension is proposed. It detects PUE attack by identifying the modulation type of the received signal using SVM classifier. Sevcik fractal dimension in frequency domain (SFDF) and Higuchi fractal dimension (HFD) of the received signal are adopted as the characteristics for classification by SVM classifier. So the task of anti-PUE attack can be carried out in spectrum sensing. Besides the parameters for spectrum sensing, i.e. SFDF and HFD, no other parameter is required to be calculated, which will decrease the calculation amount and calculation time. The Numerical results show that, the proposed method can effectively detect the PUE attack. When SNR is larger than $10 \mathrm{~dB}$, its PUE detection probability can reach 1. Even when SNR is low to -10 dB, the PUE detection probability is larger than 0.97 .
\end{abstract}

Keywords: spectrum sensing, primary user emulation attack, fractal dimension, modulation identification

\section{Introduction}

As a promising technology to alleviate spectrum scarcity, cognitive radio (CR) has been widely studied to achieve the secondary utilization of the spectrum resources. Reliable and fast spectrum sensing is a crucial technical challenge for CR realization ${ }^{[1]}$. Along with the spectrum sensing, a lot of security problems have been raised. There may be some malicious users attaching malicious attack in CR network. Some of them will send the signal emulating that of the primary user (PU) on the sensing channel, which will lead other secondary users (SUs) to failure to use the vacant spectrum resource. That is Primary user emulation (PUE) attack ${ }^{[2]}$. Distinguishing the signal of the PU from that of attacker is the key problem to defend the PUE attack. Judging whether the modulation type of the received signal is the same with that of PU is an effective way to distinguish them.

With regard to identification of digital modulation types, a automatic identification method of digital modulation types base on decision theory is proposed in [3]. It calculates five parameters of signal and uses a set of decision criteria for identifying modulation types. But it is required to calculate too many parameters causing large calculation amount. Furthermore, its identification rate is very low. A improved method of digital signals modulation identification base on decision theory is proposed in [4], but the number of parameters which are required to be calculated for modulation identification is still large, and the identification rate is still too low to detect PUE attack. In [5], they propose a cooperative automatic modulation recognition method. But five parameters are required to be calculated. The calculation amount is still large. In [6], fractal dimention is used to modulation identification in spectrum sensing. They 
use the mean and the variance of information dimension of the received signal as the characteristic to identify the modulation type. But the identification rate is still too low to require the demand of detecting PUE attack. In [7], the fractal dimensions and the Lempel-Zivcomplexity of the received signal after Hilbert-Huang Transform are calculated to extract the feature parameters for modulation identification. But due to using of artificial neural network, it needs large amount of calculation and is vulnerable to local optimum problem. And its identification rate is still low. Furthermore, for all of the methods mentioned above, more parameters other than necessary one for spectrum sensing are required to be calculated, which will cause more calculation amount and increase calculation time.

Sevcik fractal dimension (SFD) and Higuchi fractal dimension (HFD) are typical methods to calculate the fractal dimension of wavefrom ${ }^{[8]}$. In $[9,10]$, SFD in frequency domain (SFDF) and HFD are used in spectrum sensing, which obtain good results. In this paper, an anti-PUE attack method in spectrum sensing based on fractal dimension is proposed. It detects the PUE attack by modulation identification of the received signal according to its SFDF and HFD. For the proposed method, the task of anti-PUE attack can be carried out in spectrum sensing. Besides the parameters for spectrum sensing, i.e., SFDF and HFD, no other parameter is required to be calculated, which will decrease the calculation amount and calculation time.

\section{System Model}

In a CR network scene, there are three types of nodes, which are PU, SU and PUE attacker. The PU has the priority to utilize the licensed spectrum. The SU senses the spectrum periodically to seek for transmission opportunities in the white spaces. The PUE attacker launches PUE attack. It transmit fake PU signal in the special spectrum causing CR network failure to use the spectrum. At the same time, only one PUE attacker launches PUE attack, and a licensed spectrum band is only used by one PU. We assume that, the CR network knows the modulation type of PU, and the modulation type of PUE attack is different from that of PU. So the CR network can detect the PUE attack by identifying the modulation type of the received signal. We consider white Gaussian noise situation. Every SU can accurately calculate the local signal to noise ratio (SNR). To identify the modulation type of the received signal, SVM is adopted as the classifier.

To evaluate the performance of the proposed method, the detection probability of PUE $P_{d_{-} \text {PUE }}$ and the false alarm probability of PUE $P_{f_{-} \text {PUE }}$ are defined to measure the ability of the proposed method for detecting PUE attack, which can be defined as:

$$
\begin{gathered}
P_{d_{-} \mathrm{PUE}}=\frac{N_{d_{-} \mathrm{PUE}}}{N_{\mathrm{PUE}}} \\
P_{f_{-} \mathrm{PUE}}=\frac{N_{f_{-} \mathrm{PUE}}}{N_{n_{-} \mathrm{PUE}}},
\end{gathered}
$$

where $N_{\text {PUE }}$ and $N_{d_{-} \text {PUE }}$ denote the number of PUE attacks and the number of detected PUE attacks in all spectrum sensing operations, respectively. $N_{n_{-} \text {PUE }}$ and $N_{f_{-} \text {PUE }}$ denote the number of times when there is no PUE attack and the number of times when PU is mistaken for PUE attacker in all spectrum sensing operations, respectively. 


\section{Double Fractal Dimensions Characteristics Combined with SFDF and HDF}

\subsection{SFD}

Sevcik fractal dimension (SFD) derives from the calculation method of Hausdorff dimension ${ }^{[11]}$. It can be calculated as follows.

The waveform signal with a length of $N$ is composed by a set of points which are denoted by $\left(x_{i}, y_{i}\right)$, where $0 \leq i \leq N-1$. Those points are firstly normalized. The normalized points $\left(x_{i}^{*}, y_{i}^{*}\right)$ can be expressed by

$$
\begin{aligned}
& x_{i}^{*}=\frac{x_{i}}{x_{\max }} \\
& y_{i}^{*}=\frac{y_{i}-y_{\text {min }}}{y_{\text {max }}-y_{\text {min }}},
\end{aligned}
$$

where $x_{\max }$ is the maximum $x_{i}$, and $y_{\max }$ and $y_{\min }$ are the maximum and minimum $y_{i}$, respectively. $\mathrm{s}$

The SFD $D$ can be obtained by

$D=1+\frac{\ln (L)+\ln (2)}{\ln [2 \times(N-1)]}$

where $L$ is the length of the waveform which can be calculate by

$L=\sum_{i=0}^{N-2} \sqrt{\left(y_{i+1}^{*}-y_{i}^{*}\right)^{2}+\left(x_{i+1}^{*}-x_{i}^{*}\right)^{2}}$.

For communication signal, the original value of the abscissa $x$ satisfies $x=0,1,2, \ldots, N-1, x_{\max }=N-1$, and $x_{i+1}^{*}-x_{i}^{*}=1 /(N-1)$. So the formula (3) and the formula (6) can be translated to

$$
x_{i}^{*}=\frac{i}{N-1}
$$

$$
L=\sum_{i=0}^{N-2} \sqrt{\left(y_{i+1}^{*}-y_{i}^{*}\right)^{2}+\frac{1}{(N-1)^{2}}}
$$

\subsection{SFDF}

SFDF is proposed in [9], which is used in spectrum sensing and shows excellent sensing performance. It can be calculate as follows.

Firstly, for the received signal sequence $y(n)$, we apply $N$ points discrete Fourier transform (DFT) to it, and get the new sequence denoting by $\mathbf{Y}(k)$, where $n=0,1,2, \ldots, N-1$ and $k=0,1,2, \ldots, N-1$. Let $Y(k)$ to be the modulus of $\mathbf{Y}(k)$. 
Secondly, normalize $y(n)$ according to formula (4). Let $Y_{\min }$ and $Y_{\max }$ to be the minimum and maximum of $Y(k)$. Because $\mathbf{Y}(k)$ is the DFT of $y(n), Y_{\min }$ can be set to be 0 . So the normalized $Y(k)$ denoted by $Y^{*}(k)$ can be expressed as:

$Y^{*}(k)=\frac{Y(k)-Y_{\min }}{Y_{\max }-Y_{\min }}=\frac{Y(k)}{Y_{\max }}$

Then, the length of the waveform $L$ can be obtained by:

$$
L=\sum_{k=0}^{N-2} \sqrt{\left[Y^{*}(k+1)-Y^{*}(k)\right]^{2}+\frac{1}{(N-1)^{2}}} .
$$

Finally, the SFDF value of $y(n)$ expressed as $D_{S}$ can be calculated according to formula (5).

In [9], SFDF is used in spectrum sensing. They compare the SFDF of the received signal with the threshold $\lambda$ to obtain the absent or present of PU.

$$
\begin{cases}D_{S}>\lambda, & \text { PU is absent } \\ D_{S} \leq \lambda, & \text { PU is present }\end{cases}
$$

\subsection{HFD}

Higuchi fractal dimension proposed by Higuchi in 1988 is a low computational calculation method of fractal dimension ${ }^{[12]}$. It can be defined as follows.

For a discrete time sequence $y(n)$ with the length of $N$, where $n=1,2, \ldots, N, k$ new sequences are constructed firstly. The $m$ th new sequence $y_{m}^{k}$ can be constructed according to:

$$
y_{m}^{k}=\left\{y(m), y(m+k), y(m+2 k), \ldots, y\left(m+\left\lfloor\frac{N-m}{k}\right\rfloor k\right)\right\}, m=1,2, \ldots, k
$$

where $m$ is initial time, $k$ is called interval time which denotes the interval between two nodes, both $m$ and $k$ are integers. $\lfloor\cdot\rfloor$ indicates rounding down.

Then, the average curve length of constructed sequence $y_{m}^{k}$ denoted by $L_{m}(k)$ can be defined as:

$$
L_{m}(k)=\left\{\left(\sum_{i=1}^{\left\lfloor\frac{N-m}{k}\right\rfloor}|x(m+i k)-x(m+(i-1) k)|\left[\frac{N-1}{\left\lfloor\frac{N-m}{k}\right\rfloor \cdot k}\right\} / k\right.\right.
$$


where $\frac{N-1}{\left[\frac{N-1-m}{k}\right\rfloor k}$ represents the normalization factor for the curve length.

For each $k$ value, calculate the mean of $L_{m}(k)$ over different $m$ values, where $k=1,2, \ldots, k_{\max }$ and $m=1,2, \ldots, k$, and define it as the length of the curve for the time interval $k$, which is denoted by $L(k)$.

$L(k)=\frac{\sum_{m=1}^{k} L_{m}(k)}{k}$

If $L(k) \propto k^{-D_{H}}$, then $D_{H}$ is the fractal dimension of the discrete time sequence $y(n)$. If we construct a curve of which the abscissa is $\ln (1 / k)$ and the ordinate is $\ln (L(k))$, the slope of the curve can be regarded as the approximate value of $D_{H}$, which can be obtained by curve-fitting using least square method.

In [10], a spectrum sensing method based on HFD is proposed. It compares the HFD of the received signal with the threshold $\lambda$ to obtain the absent or present of PU.

$\begin{cases}D_{H}>\lambda, & \text { PU is absent } \\ D_{H} \leq \lambda, & \text { PU is present }\end{cases}$

\subsection{Double Fractal Dimensions Characteristics}

We combine SFDF and HDF to be the double fractal dimensions characteristics for SVM classifier to identify the modulation of the received signal. To analyze the double fractal dimensions characteristics of different types of signals, we take 2ASK, 2FSK, 2PSK, 4ASK, 4FSK and 4PSK for example. The simulation parameters are set as follows. The carrier frequency is set to be $200 \mathrm{MHz}$. The sampling frequency is set to be $1200 \mathrm{MHz}$. The symbol rate is $50 \mathrm{Mbps}$. The amplitude of the carrier and the frequency offset are assigned the value 1 and $50 \mathrm{MHz}$, respectively. The modulating digital symbol sequence with length of 500 is derived from independent random numbers to increase the degree of realism.

Figure 1 shows the double fractal dimension characteristics combined with SFDF and HDF of signals with different modulation types. For each modulation type, 40 signals are randomly generated and analyzed. SNR is set to be $5 \mathrm{~dB}$. It can be seen from Figure 1 that all the modulation types are easily to be identified according to their double fractal dimension characteristics combined with SFDF and HDF. 


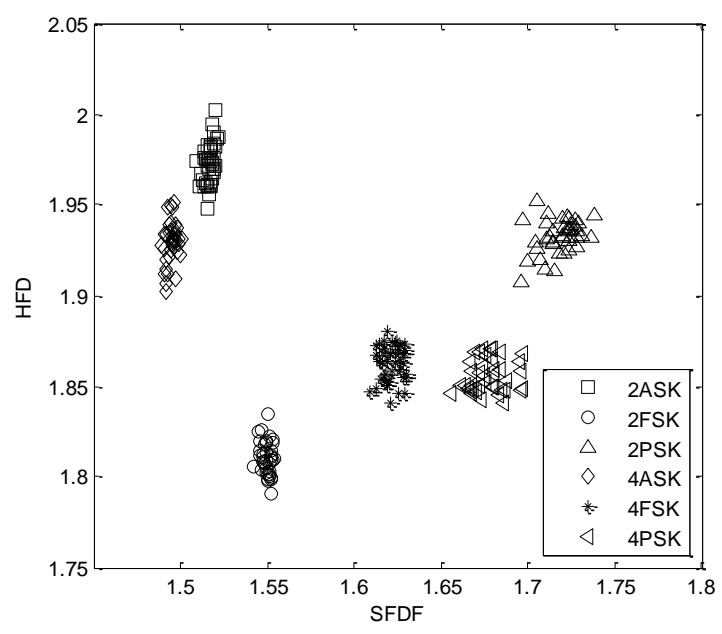

Figure 1. The Double Fractal Dimension Characteristics of Signals with Different Modulation Types

\section{Anti-PUE Attack Base on Fractal Dimension in Spectrum Sensing}

Figure 2 shows the flowchart of anti-PUE attack method in spectrum sensing based on fractal dimension. It mainly contains three parts.

a. SVM Training Part

To train the SVM classifier, some different modulation types of signals which maybe be sent by PU and PUE attacker are built. Then, calculate the SFDF values and HFD values of those signals and input them to SVM trainer to obtain the classifier model over different SNR values. To improve the identification probability, the SFDF and HFD can be obtained by averaging $k$ times sensing results.

b. Spectrum Sensing Part 


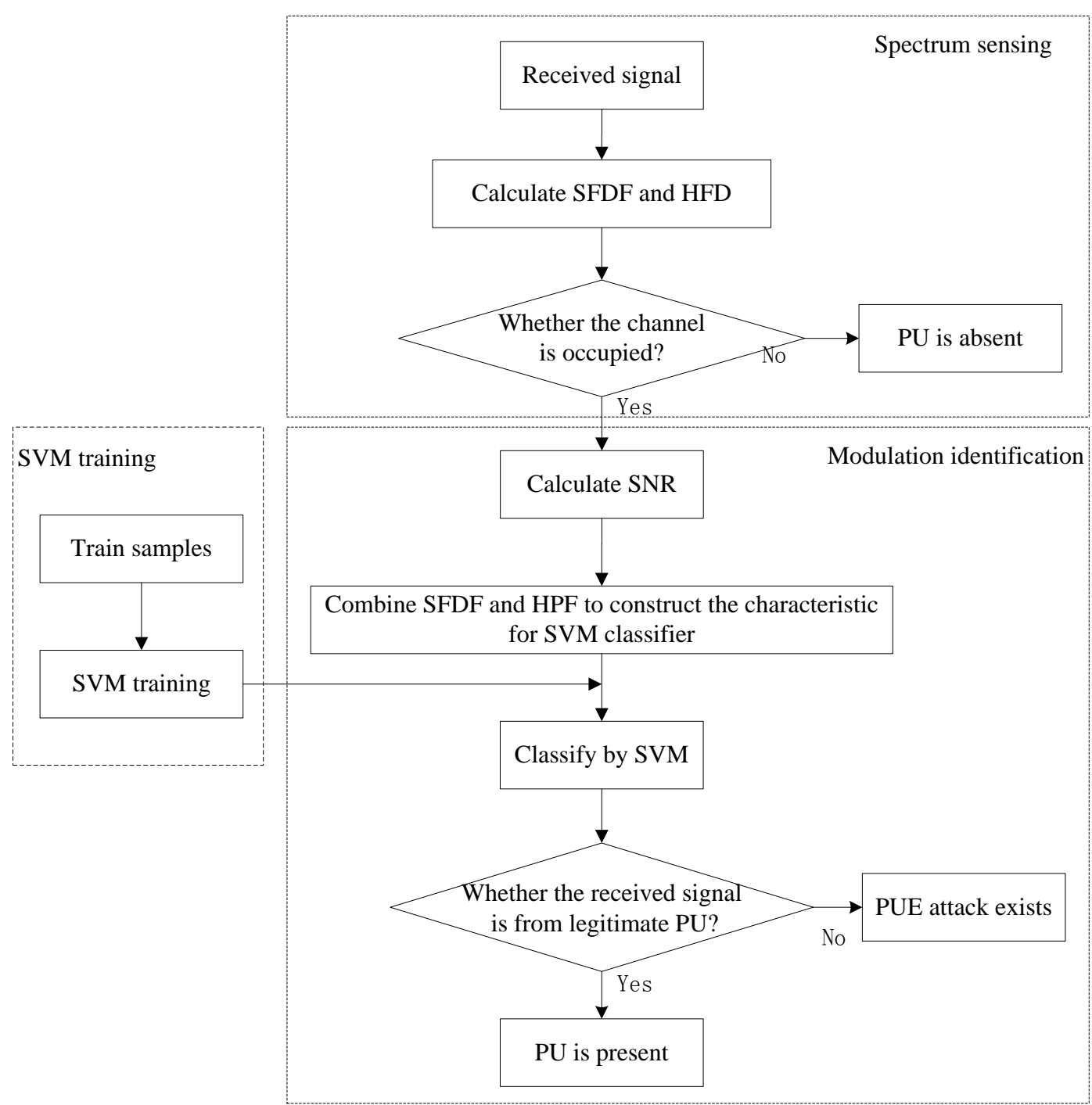

Figure 2. Flowchart of Anti-PUE Attack Method in Spectrum Sensing based on Fractal Dimension

In spectrum sensing, $\mathrm{SU}$ senses the spectrum by spectrum sensing method based on SFDF ${ }^{[9]}$ or spectrum sensing method based on HFD ${ }^{[10]}$, or both of them. If both of them are used, the sensing results of the two methods can be combined by OR rule, AND rule, and so on. If the final sensing result is that the sensed spectrum is vacant, the spectrum can be utilized by CR network. Otherwise, we have to identify the modulation type of the received signal to judge whether the received signal is from PU or PUE attacker.

\section{c. Modulation Identification Part}

Calculate the local SNR first. And then, according to the calculated SNR, select the corresponding classifier model for SVM classifier. Finally, let SFDF and HFD of the received signal as the characteristic parameter of SVM classifier, and sent them to SVM classifier to identify the modulation type of the received signal. If its modulation type is same with that of PU, the signal is judged to be from PU, otherwise is judged to be from PUE attacker, i.e. the PUE attack exists.

Furthermore, the SFDF and HFD all can be used to sense the spectrum directly ${ }^{[9,10]}$. So for the proposed method, besides the parameters for spectrum sensing, i.e. SFDF 
and HFD, no other parameter is required to be calculated. It decreases the calculation amount and calculation time.

\section{Numerical Results}

In this section, we take 2ASK, 2FSK, 2PSK, 4ASK, 4FSK and 4PSK for example to demonstrate the performance of the proposed method. In the simulations, the parameters are same with that described in Section 3.4. All numerical results are obtained by 10000 Monte Carlo simulations. For each modulation type of signal, 40 samples are trained in SVM classier. The proposed method is compared with the traditional decision theory based method ${ }^{[3]}$ and the improved decision theory based $\operatorname{method}^{[4]}$.

Figure 3 shows the dependence of the identification probability on the SNR. To analyze the effect of $k$ on the identification probability, the proposed method is simulated when $k=1, k=2, k=5$ and $k=10$, respectively. From Figure 3, it can be seen that the proposed method can get higher identification probability than the traditional decision theory based method and the improved decision theory based method. The identification probability increases with the increase of $k$. we can see from Figure 3 that, for the proposed method, when $k=1$, the identification probabilities of 2ASK and 4ASK are slightly lower than those of improved decision theory based method. But when $k$ is larger than 1 , the identification probabilities of all modulation types are all higher than those of the traditional decision theory based method and the improved decision theory based method. But the large $k$ will increase the calculation amount. So the selection of $k$ depends on the actual requirement.

Figure 4 shows the dependence of the PUE detection probability $P_{d \_ \text {PUE }}$ and the PUE false alarm probability $P_{f_{\mathrm{P}} \text { PUE }}$ on SNR over different $k$ values. The modulation types of the signal sending by PU and PUE attacker are randomly selected form 2ASK, 2FSK, 2PSK, 4ASK, 4FSK and 4PSK. From Figure 4(a), we observe that, the PUE detection probabilities are high, which means that the proposed method can effectively detect the PUE attack. Even when SNR is low to $-10 \mathrm{~dB}, P_{d_{-} \text {PUE }}$ is more than 0.97. We also notice that $P_{d \_ \text {PUE }}$ increases with the increase of SNR and $k$. When SNR is larger than $10 \mathrm{~dB}$, the PUE detection probabilities for all $k$ values can reach 1. From Figure 4(b), we can see that, the PUE false alarm probabilities are low. Even when SNR is low to $-10 \mathrm{~dB}, P_{f_{-} \mathrm{PUE}}$ is still lower than 0.14 . We also notice that $P_{f_{-} \mathrm{PUE}}$ decreases with the increase of SNR and $k$. When SNR is larger than $10 \mathrm{~dB}$, the PUE false alarm probabilities for all $k$ values approach or are equal to 0 . Larger $k$ can improve the performance of the proposed method, but at the same time, the calculation amount increases. 


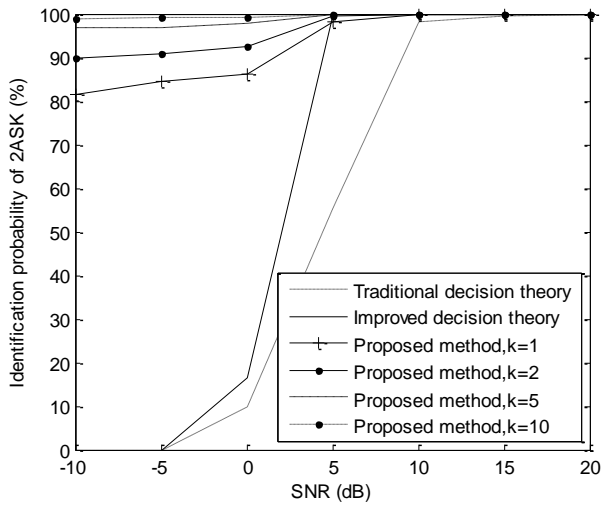

(a) $2 \mathrm{ASK}$

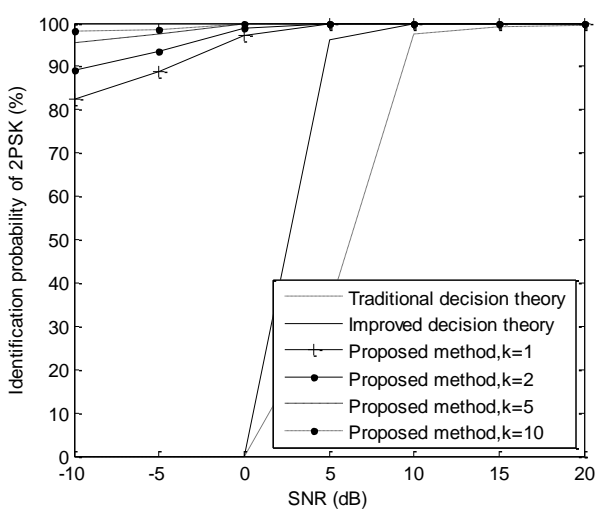

(c) $2 \mathrm{PSK}$

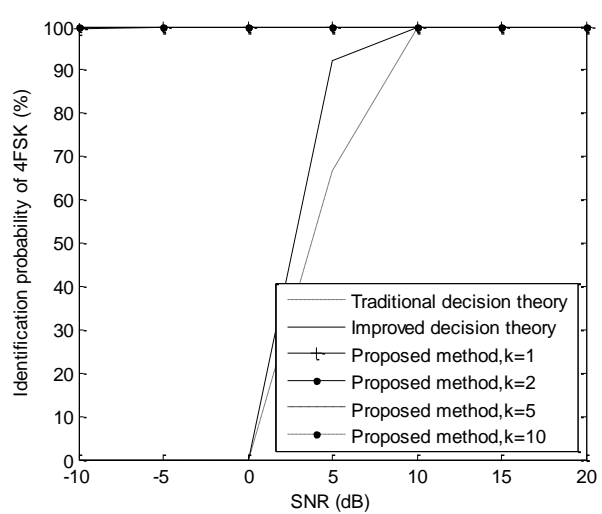

(e) 4FSK

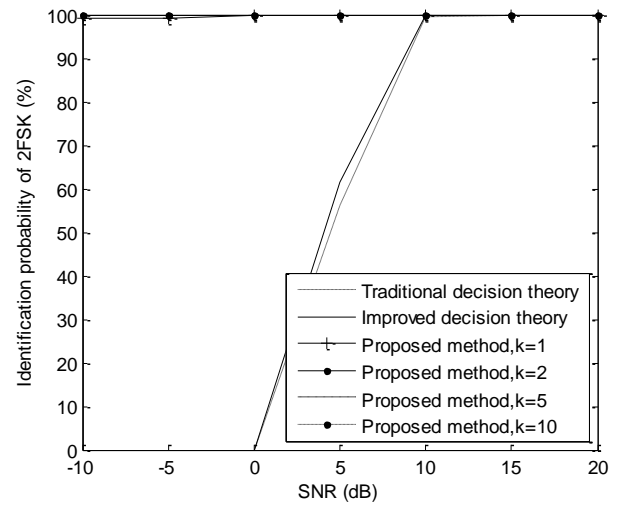

(b) $2 \mathrm{FSK}$

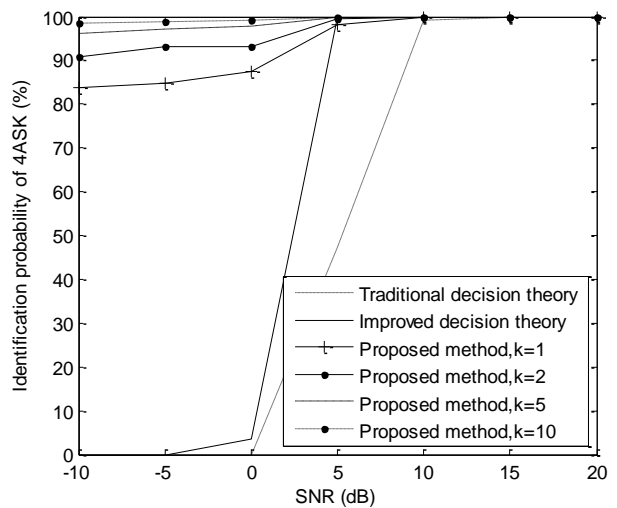

(d) $4 \mathrm{ASK}$

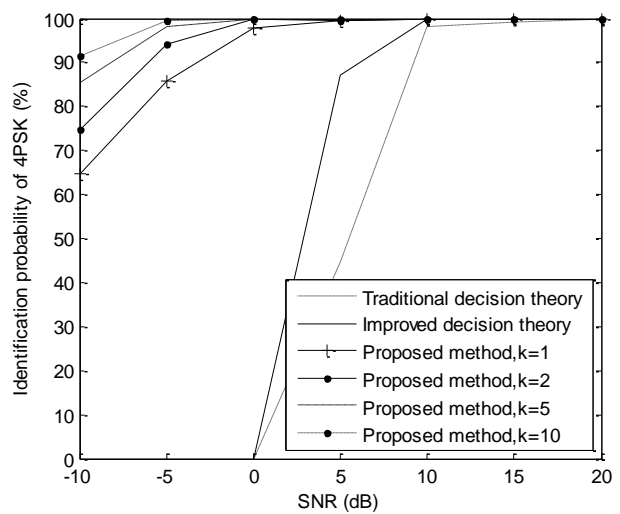

(f) 4PSK

Figure 3. Dependence of the Identification Probability on the SNR 


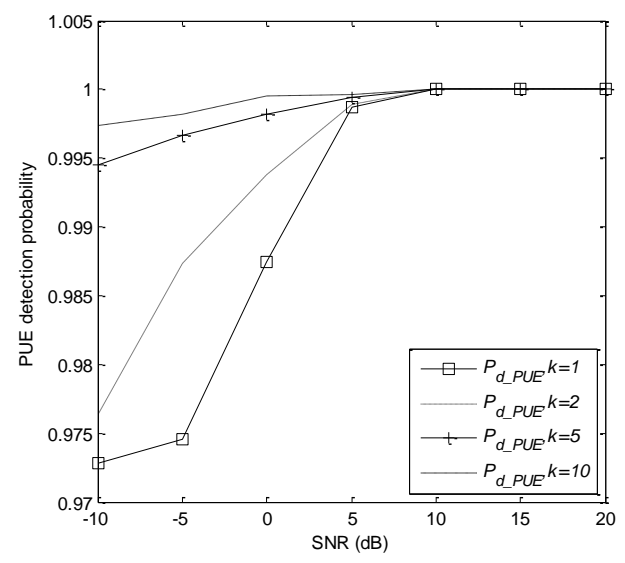

(a) PUE detection probability

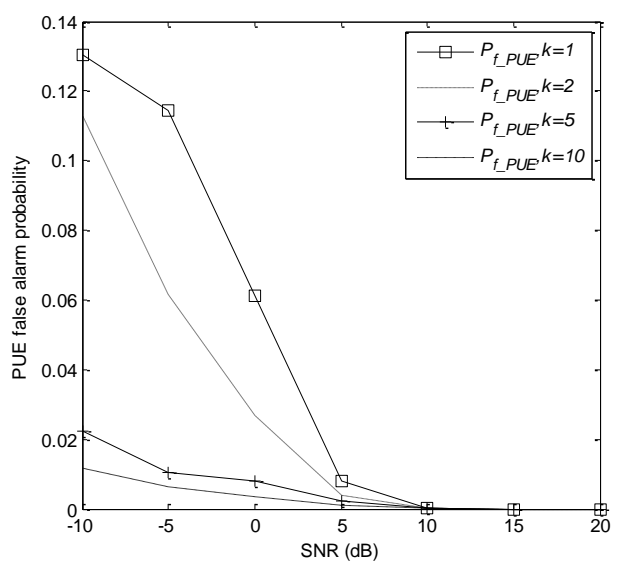

(b) PUE false alarm probability

Figure 4. Dependence of the PUE Detection Probability and the PUE False Alarm Probability on SNR

\section{Conclusion}

In this paper, an anti-PUE attack method in spectrum sensing based on fractal dimension is proposed. It detects PUE attack by identifying the modulation type of the received signal using SVM classifier. Sevcik fractal dimension in frequency domain (SFDF) and Higuchi fractal dimension (HFD) of the received signal are adopted as the characteristics for classification by SVM classifier. The Numerical results show that, the proposed method can effectively identify the digital modulation type and detect the PUE attack. Furthermore, For the proposed method, the task of anti-PUE attack can be carried out in spectrum sensing. Besides the parameters for spectrum sensing, i.e., SFDF and HFD, no other parameter is required to be calculated, which will decrease the calculation amount and calculation time.

\section{Acknowledgements}

This paper is funded by the Science Foundation of Heilongjiang Province for the Youth (No.QC2015070) and the Scientific Research Foundation for Doctor of Heilongjiang Bayi Agricultural University (No. XDB2015-28, No. XYB2013-23).

\section{References}

[1] H. Sun, A. Nallanathan, C.-X. Wang and Y. Chen, "Wideband spectrum sensing for cognitive radio networks: a survey", IEEE Wireless Communications, vol. 20, (2013), pp. 74-81.

[2] A. G. Fragkiadakis, E. Z. Tragos and I. G. Askoxylakis, "A survey on security threats and detection techniques in cognitive radio networks", IEEE Communications Surveys \& Tutorials, vol. 15, (2013), pp. 428-445.

[3] E. E. Azzouz and A. K. Nandi, "Automatic identification of digital modulation types", Signal Processing, vol. 47, (1995), pp. 55-69.

[4] D. Zhang and X. Wang, "Improved method of digital signal modulation identification based on decision theory", Journal of Computer Applications, vol. 29, (2009), pp. 3227-3230.

[5] C. Mei and Z. Qi, "Cooperative automatic modulation recognition in cognitive radio[J]. The Journal of China Universities of Posts and Telecommunications, 2010, (2)", The Journal of China Universities of Posts and Telecommunications, vol. 17, (2010), pp. 46-52.

[6] C. Xiaobo, C. Hong and C. Yin, "Spectrum sensing for cognitive ultra-wideband based on fractal dimensions", in 2011 Fourth International Workshop on Chaos-Fractals Theories and Applications (IWCFTA), Hangzhou, China, (2011).

[7] S. Zhao, L. Qin and X. Chen, "Digital modulation recognition based on complexity measure", Computer Engineering and Applications vol. 51, (2015), pp. 226-231. 
[8] R. Esteller, G. Vachtsevanos, J. Echauz and B. Litt, "A comparison of waveform fractal dimension algorithms", IEEE Transactions on Circuits and Systems I: Fundamental Theory and Applications, vol. 48, (2001), pp. 177-183.

[9] S. Fu, Y. Li, F. Ye and Z. Gao, "Fast blind spectrum sensing using Sevcik fractal dimension in frequency", Journal of Jilin University (Engineering and Technology Edition), vol. 44, (2014), pp. 854-860.

[10] S. B. Kordan and B. Abolhassani, "Blind spectrum sensing for cognitive radio based on complexity measurement", in 2011 19th Iranian Conference on Electrical Engineering (ICEE), Tehran, (2011).

[11] C. Sevcik, "A procedure to estimate the fractal dimension of waveforms", Complexity International, vol. 5, (1998), pp. 1-19.

[12] T. Higuchi, "Approach to an irregular time series on the basis of the fractal theory", Physica D: Nonlinear Phenomena, vol. 31, (1988), pp. 277-283. 
International Journal of Security and Its Applications

Vol. 10, No. 10 (2016) 